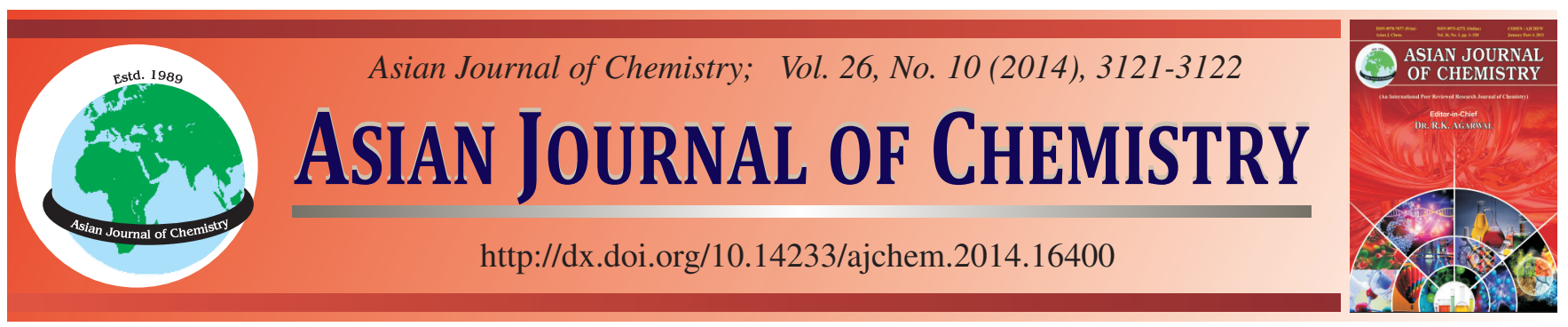

NOTE

\title{
Determination of Vanillin in Milk Powder by Rapid Column High Performance Liquid Chromatography
}

\author{
Cheng $\mathrm{Li}^{1}$ and Chang-Li ZhaO ${ }^{2, *}$
}

${ }^{1}$ Jiangxi Normal University, Nanchang 330022, P.R. China

${ }^{2}$ School of Education, Nanchang University, Nanchang 330031, P.R. China

*Corresponding author: E-mail: jbcai@ustc.edu.cn

Received: 20 September 2013;

Accepted: 9 December 2013;

Published online: 10 May 2014;

AJC-15194

A rapid column high performance liquid chromatography method for the determination of vanillin in milk powder was studied. The vanillin was extracted from the sample with $40 \%$ methanol by the ultrasonic extraction and then was purified by solid phase extraction with $\mathrm{C}_{18}$ cartridge. Vanillin was separated on a ZORBAX stable bound $(4.6 \times 50 \mathrm{~mm}, 1.8 \mathrm{~mm})$ rapid chromatographic column with watermethonal (65:35) as mobile phase at a flow-rate of $2 \mathrm{~mL} / \mathrm{min}$ and monitored with the photodiode array detector at $309 \mathrm{~nm}$. The recoveries of the vinillin are $98-103 \%$ and the relative standard deviations are $0.46-0.58 \%$. This method was applied to the determination of vanillin in milk powder with good results.

Keywords: High performance liquid chromatography, Vanillin, Milk powder.

Food additives monitoring was an important part of food safety monitoring. Vanillin, the world's largest production and consuming synthetic fragrances, was harmful when adding too much so that the determination of vanillin in food had important significance ${ }^{1}$. Liquid chromatography was widely used method for the determination of vanillin, but the ordinary high-performance liquid chromatography always take too much time (about $10 \mathrm{~min})^{2-7}$. To reduce the analysis time, we studied the HPLC with fast separation column to analyze the vanillin from milk powder. The vanillin in the samples can separate baseline in only 1 min using this method, which was much faster than conventional liquid chromatography analysis.

Agilent 1100 HPLC, including: HP 1100 quaternary pump, HP1100 UV diode array detector, HP 1100 autosampler and Chem Station chromatography workstation.

Methanol (HPLC, produced by the fisher company); Vanillin (purity of at least $99 \%$, produced by the SIGMA company); Double distilled water (produced by the Milli-Q50 instrument of USA Millipore Corporation, which the resistance could get $18 \mathrm{M} \Omega \mathrm{cm}$ or above) and the rest of reagents were analytical grade or above.

Chromatographic conditions: Components were separated on a ZORBAX Stable Bound $(4.6 \times 50 \mathrm{~mm}, 1.8 \mathrm{~mm})$. The mobile phase was $35 \%$ percents of methanol at a flow rate of $2 \mathrm{~mL} / \mathrm{min}$. Sample injection volume was $5 \mathrm{~mL}$ and the detection wavelength was $309 \mathrm{~nm}$. The chromatogram of sample and standard sample in the above chromatographic conditions were shown in Fig. 1.

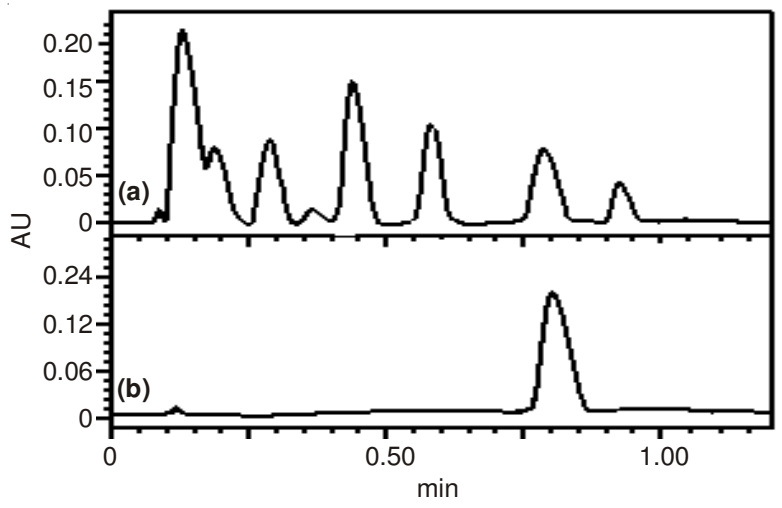

Fig. 1. Chromatograms of vanillin (b) and milk sample (a)

Sample preparation: $2 \mathrm{~g}$ sample was withdrawn into a $100 \mathrm{~mL}$ beaker add $40 \mathrm{~mL} 40 \%$ methanol and then was ultrasonically extracted for $10 \mathrm{~min}$ at $40{ }^{\circ} \mathrm{C}$. After the beaker cooled down, the liquid was withdrawn into a flask with $40 \%$ methanol to $50 \mathrm{~mL}$. With $5 \mathrm{~mL}$ solution through the preactivation Waters Sep-Pak-C18 solid phase extraction column at a flow rate $10 \mathrm{~mL} / \mathrm{min}$, discard the first $3 \mathrm{~mL}$ solution and then filter the rest of $2 \mathrm{~mL}$ solution collected with $0.45 \mathrm{~mm}$ syringe filters. The injection volume was $5 \mathrm{~mL}$. 
Water and methanol were chosen as the mobile phase for separating vanillin. When the ratio of water and methanol was $35: 65$, the vanillin in the samples could be completely separated from the other coexisting components. As we know, conventional high performance liquid phase separation column needed a long time to separate vanillin, so we chose the ZORBAX Stable Bound $(4.6 \times 50 \mathrm{~mm}, 1.8 \mathrm{~mm}) \mathrm{C}_{18}$ fast separation column to short the experiment time in this study. With this column, the vanillin could be separated in 1 min which was $85 \%$ faster than the conventional column.

The spectra of each vanillin could be found between 200$400 \mathrm{~nm}$ wavelengths by the UV diode array detector and vanillin had a greater absorption and the less background interference at $309 \mathrm{~nm}$, so we chose the $309 \mathrm{~nm}$ wavelength as the experiment condition. The vanillin of the sample was identified by the retention time, UV spectra and standard sample. In addition, we also analyzed the peak purity to confirm that the vanillin was completely separated from the coexistence components of the sample.

Sample preparation: Vanillin had a greater solubility in aqueous methanol solution and methanol concentration of $40 \%$ was acceptable. With $40 \mathrm{~mL} 40 \%$ aqueous methanol solution and ultrasonic oscillation $10 \mathrm{~min}$, vanillin in the sample could be completely dissolved in methanol. Therefore, this method was applied in sample preparation.

Milk contains vanillin, also contain oily substances. Such substances can't be completely eluted in this mobile phase conditions. As we know, oily substances would residue accumulate on $\mathrm{C}_{18}$ column, increase chromatographic system backpressure and loss of efficiency, so degreasing treatment is indispensable before experiment. So our group carried out Study on the processing method above. With a waters SPE vacuum extraction device, 20 samples simultaneously per treatment, flow rate were $10 \mathrm{~mL} / \mathrm{min}$ when extraction column activation and sample pre-separation. Used $3 \mathrm{~mL}$ of methanol for extraction column activation and $10 \mathrm{~mL}$ water for methanol washing and took $40 \%$ methanol extract of the sample pass through the extraction column so that oily substances retained in the column. Vanillin, not retained in the extraction column, could be directly determination by HPLC.

Regression equation, correlation coefficient and detection limit: Prepared series of standard solution of vanillin and the concentrations were $100,20,4,0.8 \mathrm{~g} / \mathrm{mL}$ ). According to the peak area of different concentrations, calculated the regre- ssion equation after injection. Regression equation is: $\mathrm{A}=4.18$ $\times 105 \mathrm{C}+368$ (A, the peak area; $\mathrm{C}$, the concentration; units, $\mathrm{g} / \mathrm{mL}$ ), Correlation coefficient $\mathrm{r}=0.9999$. Because the letter dry ratio $\mathrm{S} / \mathrm{N}=3$, detection limit was $10 \mathrm{mg} / \mathrm{mL}$.

Results of sample analysis: Milk samples were determined by selected chromatographic conditions after sample pretreatment. When measured, accurately weighed in duplicate, one was standard, another one was of added standard vanillin of known quantity. Each sample was measured under the same condition 5 times in parallel. Recovery was calculated with the amount of sample measured divided by the amount of added standard sample. And the relative standard deviation was calculated with the 5 times results in parallel (Table-1).

\begin{tabular}{cccc}
\multicolumn{4}{c}{ TABLE-1 } \\
RESULTS OF SAMPLE ANALYSIS \\
\hline Sample & $\begin{array}{c}\text { Measured } \\
\text { values }(\mu \mathrm{g} / \mathrm{g})\end{array}$ & $\begin{array}{c}\text { Recovery of } \\
\text { standard sample } \% \\
\text { (added amount was }\end{array}$ \\
\hline Milk-1 & 287 & 0.46 & $98 \mathrm{~g})$ \\
Milk -2 & 412 & 0.52 & 103 \\
Milk-3 & 185 & 0.48 & 99 \\
\hline
\end{tabular}

\section{Conclusion}

This method used solid-phase extraction pre-separation, rapid column high performance liquid chromatography (HPLC) method for the determination of vanillin in milk powder. Vanillin was separated within the $1.0 \mathrm{~min}$. and the recoveries of the vinillin were more than $85 \%$. The result showed that the method is simple, rapid, reliable and to a certain extent, for the rapid determination of vanillin present in milk.

\section{REFERENCES}

1. M. Baig, G. Kapse and S. Raju, Asian J. Chem., 13, 185 (2001).

2. Y. Huang, Z. Lu and G. Lu, Chinese J. Health Lab., 12, 566 (2002).

3. L. Nagpurkar, A. Chaudhari and J. Ekhe, Asian J. Chem., 14, 1387 (2002).

4. D. Reddy, B. Gurupadayya, Y. Manohara and V. Bhaskar, Asian J. Chem., 19, 2797 (2007).

5. F. Rind, U. Mughal, A. Memon, F. Almani and M. Laghari, Asian J. Chem., 21, 2849 (2009).

6. A.J. Aktas, Asian J. Chem., 22, 3719 (2010).

7. M. Chen, J. Wang, Y. Zhang and K. Meng, Asian J. Chem., 24, 1029 (2012). 